\section{Auditory and visual control over chained operants: II. Replication and effects of darkness on auditory control ${ }^{1}$}

R. M. GILBERT, Addiction Research Foundation, Tororito 179, Ont., Canada, and P. G. CROSKERRY, McMaster University, Hamilton, Ont, Canada

Hooded rats trained to emit a two-operant chain under auditory control were again found to be less affected by omission of the signal correlated with the second operant than rats treated similarly but under visual control. Auditory control faded in darkness too, showing that control was not necessarily assumed by unscheduled visual sources.

Gilbert \& Moore (1967) reported that control by an auditory difference over the later component of a two-operant chain was lost more rapidly than control by a visual difference. At least two objections can be raised against that conclusion: (1) The tests for discriminative control did not provide evidence for auditory control at any stage of the study. The only reported evidence for auditory control was in the description of the training procedure. The auditory difference may never have participated in the control of the chain. (2) The visual signals used may have been far more discriminable from their respective absences than the auditory signal, which was a crude $400-\mathrm{Hz}$ tone. It appears that rats may be insensitive to frequencies below about $1000 \mathrm{~Hz}$ (Munn, 1950), and thus it is possible that only harmonics and other low-intensity, nondominant characteristics of the tone were important. Hence the difference found between the signals might not indicate a difference between modalities but only a difference between particular signals.

One objective of the present study was to provide for replication of the earlier finding; another was to attempt to answer the above objections by testing for control soon after training and by using a high-intensity white noise as the auditory signal.

In addition, the present study was designed to investigate one possible source of control when the auditory control had faded. The common assumption that the control of simple sequences of behavior becomes "internalized" with practice makes proprioceptive events the most obvious candidate for the assumption of control. However, in an illuminated chamber, visual sources are available: the animal could come to pull the trapeze in such a way as to finish facing the lever, and light reflected from the lever could provide the occasion for approaching and pressing it. Some rats in the present study were trained and tested in darkness using an auditory signal.

\section{SUBJECTS}

Nine naive male black-hooded rats were used, aged 3 months at the beginning of a 1 -month deprivation program which stabilized body weights at $80 \pm 2 \%$ of their weights when fed freely at 3 months of age. APPARATUS

Two Foringer Type 1107 chambers were used. In the ceiling of Chamber 1 were a 1134B stimulus lamp and a 1107CL trapeze, centrally mounted and respectively $320 \mathrm{~mm}$

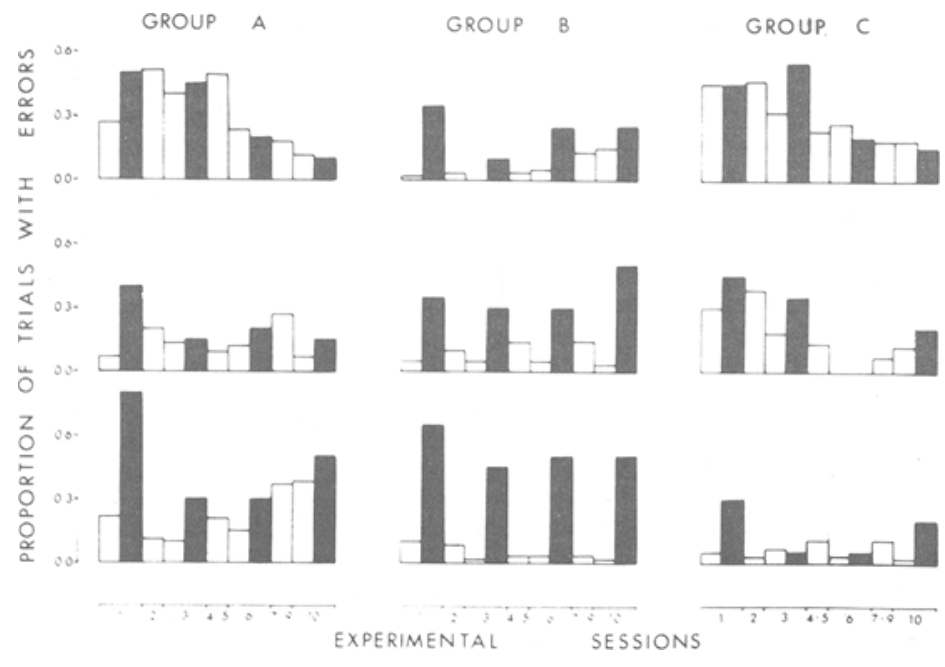

and $110 \mathrm{~mm}$ from the function panel. A similar trapeze was mounted centrally $180 \mathrm{~mm}$ from the function panel in the ceiling of Chamber 2; this trapeze was the input of a proximity detector of adjustable sensitivity. Each box had a lever mounted in the left-hand position (initially absent), a speaker behind the lever, a $1107 \mathrm{HL}$ houselight on the function panel (disconnected in Chamber 2), and a Type 1284 dispenser which delivered one $45-\mathrm{mg}$ Noys pellet on command. The chambers were set in ventilated sound-resistant and light-proof housing together in a cubicle in the room containing the electromechanical programming circuitry. Data were recorded on electromechanical counters and Rustrak chart recorders.

\section{PROCEDURE}

After deprivation, each rat was allocated to one of three equal groups. Groups $A$ and $B$ were trained and tested in Chamber 1. For these six rats delivery of a food pellet was made conditional upon pressing the lever and then pulling the trapeze; the groups differed only in the signal indicating the occasion for a trapeze pull, which was the light in the rear of the ceiling in the case of Group B and white noise which raised the average sound intensity from 75 to $85 \mathrm{~dB}$ in the case of Group A. The houselight was on continuously during sessions for both groups. After the animals in Groups A and B had been magazine trained, the trapeze and the respective signal were introduced, the white noise being initially at less than full intensity to avoid aversion. All six rats played with the trapeze when it was introduced and pulled it with little shaping. After a few pulls the signal was offset briefly on occasion after reinforcement, the offset durations being extended until they were about $30 \mathrm{sec}$, this being done in such a way as to minimize trapeze pulling when the signal was off. When, for a particular rat, only one trapeze pull occurred throughout 10 consecutive signal-off periods of about $30 \mathrm{sec}$ each the session was terminated. Three sessions of discrimination training were required for each rat, with not more than 200 pellets being delivered in any one session. The lever was now introduced and signal onset was made contingent upon successive approximations to lever pressing: A trapeze pull during the signal continued to be followed by food. The three rats in Group B, and one in Group A, explored the lever when it was introduced and little

Fig. 1. Proportion of trials during which the first operant was emitted at least once when the second operant would have been appropriate. Solid bars refer to test trials; open bars refer to regular trials. 
shaping was required. The first experimental session began with the first lever press. The other two rats in Group A did not press the lever within the first 50 shaping trials, but did so soon after the beginning of the next training session, whereupon the first experimental session began.

The animals in Group $\mathrm{C}$ were trained and tested in Chamber 2 to emit the sequence trapeze pull $\rightarrow$ lever press in darkness. After magazine training in low illumination, and before the lever was introduced, food delivery was made contingent upon exploration of the hole in the function panel through which the lever was to project. When the animals spent most of the time at the hole, the lever and white noise were introduced, the latter gradually, and the chamber was subsequently maintained in darkness. Each rat pressed the lever as soon as it was introduced. The signal was then removed for increasingly longer periods, in the manner of the procedure for the other groups, except that it was not possible to avoid offsetting the signal when the animal seemed likely to press because the rats were invisible. The criterion of control used for Groups A and B was recognized during the third session of discrimination training, whereupon the trapeze was introduced at the beginning of the following day's session. Onset of the signal now became contingent upon a rat's being successively closer to the trapeze, then touching it, then holding it, and then pulling it. The approximations to pulling the trapeze were assessed using the proximity detector. Each rat in Group C pulled the trapeze within 50 shaping trials and the first experimental session began with the first trapeze pull.

There were 10 experimental sessions, each of 100 trials. A regular trial consisted of a period without the signal, when emitting the first operant produced the signal, and a period with the signal, when emitting the second operant produced food, offset the signal, and initiated the next trial. Errors, i.e., emitting one operant when the other was appropriate, had no programmed effect. During a test trial the signal was not presented as a consequence of emitting the first operant in its absence, but reinforcement was still contingent upon emission of the sequence first operant $\rightarrow$ second operant. Sessions $1,3,6$, and 10 each consisted of 80 regular trials and 20 test trials. The test trials occurred in four blocks of five, beginning with the $21 \mathrm{st}, 41 \mathrm{st}, 61 \mathrm{st}$, and 81 st trials. The other six sessions consisted of 100 regular trials.

\section{RESULTS AND DISCUSSION}

If a rat's behavior was not under the control of the signal and its absence, then the behavior would be unaffected by the unavailability of the signal during test trials. On the other hand, behavior under discriminative control would be affected. In particular, because during a test trial only the occasion for emitting the first operant occurred, a rat whose behavior was under discriminative control might have been expected to continue emitting the first operant when emission of the second operant would have been followed by food delivery. Proportions of trials during which errors involving the first operant were made, during test trials and normal trials, are shown in Fig. 1. Errors following an operant by less than $0.5 \mathrm{sec}$ are not represented. The figure shows that the three rats in Group B, and most rats in Groups $A$ and $C$, were more likely to emit errors involving the first operant during the test trials of Session 1 than during the regular trials. The animals in Group B continued to exhibit this difference between regular and test trials whereas the other animals did not.

Thus the results of the earlier study were replicated in a situation in which the auditory signal was relatively more prominent, and in which the auditory signal was initially associated with the control of the chained behavior in at least half of the possible animals. In addition, auditory control did not persist in darkness, indicating that behavior initially under auditory control does not necessarily come under visual control with practice.

A significant feature of the data was that, even when control was indicated by a greater proportion of first-operandum errors during test trials, these errors were often not made on at least half of the test trials, suggesting other sources of control. To some extent this was because first-operandum errors became less likely towards the end of each block of five, suggesting blunting of control within blocks. Such blunting is supported by the distribution within blocks of errors involving the second operandum; these errors occurred inostly towards the end of a test block, a consequence of the reinforcement of the second operant in the absence of the signal.

Blunting or not, it remains true that, even considering only the first trial of each test block, the rats in Group B were not always affected by omission of the visual signal. The visual control could have been sporadic, and shared with other sources. Alternatively, the behavior may not have been under visual control and yet may have been sometimes disrupted by the nonappearance of an event which usually occurred, namely, the onset of the visual signal. The basis of the difference between the auditory and visual signals remains obscure.

REFERENCES

GILBERT R., \& MOORE, M. Auditory and visual control over chained operants. Psychonomic Science, 1967, 8, 267-268.

MUNN, N. L. Handbook of psychological research on the rat. Boston: Houghton Mifflin, 1950. NOTE

1. This study was carried out at the University of Aberdeen, Scotland, during the later part of 1967. It was supported in part by a grant from the British Medical Research Council.

\section{Noncontingent partial reinforcement reduces spontaneous alternation'}

JOEL ADKINS, ROBERT K. HILLES, DAVID S. WEISBROD, ${ }^{2}$ and MICHAEL R. EMMENS, University of Oregon, Eugene, Oreg. 97403

Two groups of 12 rats were tested for spontaneous alternation in a $T$ maze. Ss rewarded with food after each trial alternated goal arms significantly more often $(p=.011)$ than Ss for which reward was withheld randomly on one-half of the trials regardless of the animal's response. $A$ possible relation between the effects of partial reinforcement and of brain damage in the limbic system or frontal cortex was discussed. 